\title{
Improving the methodology of sample preparation of sunflower seeds when determining the content of oleic acid by NMR
}

\author{
Oleg Agafonov* and Sergey Prudnikov
}

V.S. Pustovoit All-Russian Research Institute of Oil Crops, 35003817 Filatova St., Krasnodar, Russia

\begin{abstract}
Developing modern instrumental express methods for monitoring quality parameters requires special attention to the comparability of measurement results with reference methods. This article includes the result of improving the method for determining oleic acid content in sunflower seed oil using pulsed NMR technique. In the conditions of industrial laboratories, different seed quality and temperature have the greatest influence on the accuracy of measuring oleic acid content. It is possible to reduce the influence of different quality by increasing the number of simultaneously analyzed samples. A method for preliminary SHF treatment of seeds was proposed that could significantly reduce the time of seed sample preparation with the temperature below $15^{\circ} \mathrm{C}$. Due to the special characteristics of SHF treatment, seeds are heated under certain parameters to the temperature of $20-25^{\circ} \mathrm{C}$ without loss of moisture contained in the analyzed sample what is especially important for the comprehensive determination of quality parameters of sunflower seeds using pulsed NMR method.

To eliminate temperature influence, it was proposed to measure the temperature of each analyzed sample before the analysis. This allowed significant increasing the accuracy of measuring oleic acid content. These solutions were implemented in a sampling device that made a sample with a volume of $25 \mathrm{~cm}^{3}$ and at the same time measured its temperature.
\end{abstract}

\section{Introduction}

As a result of successful work of domestic and foreign plant selection breeders, new varieties and hybrids of oilseeds appear that differ in a good way from traditional ones in their properties.

One of the examples of this kind is oilseeds with modified fatty acid composition of oil: high-oleic sunflower and rapeseed, low-linolenic flax, non-erucic rape. Changes in the fatty acid composition of seed oil changes its functional and technological properties, nutritional and energy value $[1,2]$.

Increased content of oleic acid (more than $75 \%$ ) increases shelf life of oil up to 4 times. Such oil is an excellent raw material for the production of margarines and spreads, as the technological processes of their production become simplified. High-oleic oils can be used for a long-time frying what makes them optimal for use as frying oils [3]. In terms of oleic

*Corresponding author: Sacred_jktu@bk.ru 
acid, high-oleic sunflower oil is superior to olive oil while it is much cheaper. It is also important that sunflower oil is traditional in Russia what makes it more attractive to consumers.

Depending on the method of commercial batch formation, growing conditions and natural characteristics of seeds, oleic acid content in oilseeds can vary significantly [4]. As a result, its technological properties and price also change.

Given this, the factories involved in the procurement and processing of oilseeds get a challenge on introducing new ways for monitoring quality parameters. Implemented methods for monitoring quality parameters should meet the following requirements: being easy implemented, having high accuracy, excluding the use of chemicals and allowing quick measurements.

Methods for defining quality parameters based on pulsed NMR technique are fully consistent with these requirements. They are quick, non-destructive, easy to implement, do not require using toxic organic solvents, do not depend on the special characteristics of operator, require no complex sample preparation. These methods allow determining both the quantitative and qualitative composition of oilseeds.

The authors from the All-Russian Scientific Research Institute of Oilseeds, (Krasnodar) proposed a comprehensive system for assessing the quality of sunflower seeds using pulsed NMR method. Developed system is quick, non-destructive and allows simultaneous defining oil content, moisture and oleic acid content in sunflower seed oil. Moreover, it can be implemented on modernized AMB-1006M NMR analyzers that are already in operation what significantly reduces implementation costs [5-7].

Developed system is currently implemented at several enterprises of the oil and fat industry engaged in the procurement and processing of oilseeds. Results of production tests of this method at enterprises showed that the accuracy of determining sample temperature is greatly influenced by the accuracy of measuring oleic acid content in seed oil and by its difference from the recommended temperature for measuring $\left(23^{\circ} \mathrm{C}\right)$.

The goal of this work was to study ways to improve the accuracy of the results of defining oleic acid content in sunflower seed oil using pulsed NMR method.

\section{Materials and methods}

The studies were performed using the seeds of high-oleic sunflower grown in the Krasnodar Territory.

Oleic acid content in sunflower seed oil was determined using reference method based on chromatographic method in accordance with GOST 30418-96 "Vegetable oils. Method for determination of fatty acid content" using a chromatograph (Chromatek-Crystal 5000). In the following way: from pre-selected seeds the oils were released by pressing method, then filtered and determined its fatty acid composition using gas chromatograph [8].

The corresponding results are shown in Table 1.

Table 1. Fatty acid content in the studied samples of sunflower seeds.

\begin{tabular}{|c|c|c|c|c|c|c|c|c|c|c|}
\hline \multirow[t]{2}{*}{ Sample } & \multicolumn{10}{|c|}{ Weight content of fatty acid, $\%$} \\
\hline & $\frac{0}{0}$ & $\begin{array}{l}\frac{0}{0} \\
\stackrel{0}{\Xi}\end{array}$ & 䍘 &  & 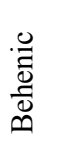 &  & 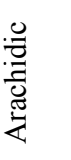 & 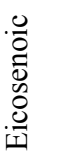 & 洂 & 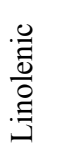 \\
\hline 1 & 83.87 & 8.07 & 3.38 & 2.81 & 0.94 & 0.27 & 0.26 & 0.24 & 0.09 & 0.07 \\
\hline 2 & 79.00 & 11.68 & 3.53 & 3.76 & 1.07 & 0.28 & 0.30 & 0.21 & 0.10 & 0.07 \\
\hline 3 & 79.57 & 11.54 & 3.61 & 3.49 & 0.95 & 0.24 & 0.28 & 0.19 & 0.08 & 0.05 \\
\hline
\end{tabular}




\begin{tabular}{|l|l|l|l|l|l|l|l|l|l|l|}
\hline 4 & 79.59 & 12.38 & 3.60 & 2.72 & 0.92 & 0.24 & 0.22 & 0.21 & 0.08 & 0.04 \\
\hline 5 & 74.76 & 16.65 & 3.76 & 3.04 & 0.94 & 0.26 & 0.25 & 0.21 & 0.08 & 0.05 \\
\hline 6 & 75.99 & 15.37 & 3.82 & 3.08 & 0.89 & 0.26 & 0.25 & 0.20 & 0.10 & 0.04 \\
\hline 7 & 79.69 & 12.75 & 3.49 & 2.46 & 0.84 & 0.21 & 0.22 & 0.21 & 0.09 & 0.04 \\
\hline 8 & 77.73 & 14.01 & 3.58 & 3.06 & 0.87 & 0.23 & 0.23 & 0.17 & 0.08 & 0.04 \\
\hline 9 & 78.40 & 13.73 & 3.60 & 2.62 & 0.88 & 0.23 & 0.22 & 0.20 & 0.08 & 0.04 \\
\hline 10 & 88.45 & 4.39 & 3.07 & 2.34 & 0.92 & 0.27 & 0.22 & 0.23 & 0.07 & 0.04 \\
\hline 11 & 82.34 & 9.40 & 3.29 & 3.22 & 0.92 & 0.27 & 0.28 & 0.17 & 0.07 & 0.04 \\
\hline 12 & 73.06 & 18.59 & 3.76 & 2.95 & 0.83 & 0.26 & 0.24 & 0.18 & 0.09 & 0.04 \\
\hline
\end{tabular}

For this study, seeds of the Oreshek variety were used. Specified data reveal that there is a significant variation in oleic acid content in seed oil within one variety - from $73.06 \%$ to $88.45 \%$. Such range of oleic acid content within one variety can be explained by different factors: biological characteristics of variety, weather conditions, growing technology and formation of seed sales batches. The data obtained on the high variation of oleic acid content in sunflower seed oil, depending on various factors, is confirmed by other researchers [9].

Defining oleic acid content using pulse NMR technique was carried out according to the developed method using AMB-1006M serial NMR analyzer (All-Russian Scientific Research Institute of Oilseeds, Russia) modernized for defining oleic acid content in sunflower seed oil. The work of the NMR analyzer is based on the impulse method of CarrParcell-Maybume-Gill, with control and processing of results on the basis of a personal computer [10]. From a sample of seeds prepared in accordance with temperature control method at the temperature of $23 \pm 0.5^{\circ} \mathrm{C}, 10$ samples with a volume of $25 \mathrm{~cm}^{3}$ were taken. Then, oleic acid content was measured for each sample. Final result included average value for 10 samples. The advantages of this method are lack of sampling preparation, operational nature (time of one analysis not more than 30 seconds), simultaneous determination of oiliness, humidity and oleic acid content in sunflower seed oil without their destruction, lack of requirements for personnel qualification.

Dosing of samples was carried out using a specially developed and patented sampling device that allowed easy sampling of a given volume [11].

An infrared pyrometer (OPTRIS MS, Germany) was used to determine the temperature of tested seeds.

\section{Result and discussion}

Developed method is based on the dependence of NM-relaxation characteristics of protons in analyzed samples on the content of oleic acid in seed oil, while the measurement of these characteristics should be carried out at the temperature of $23 \pm 0.5^{\circ} \mathrm{C}$. This is due to the fact that relaxation characteristics, namely, weighted average spin-spin relaxation time of oil protons, are significantly dependent on temperature.

In our country, harvesting and processing of sunflower seeds at oil and fat factories is carried out in autumn-winter period. As a result, seeds have a temperature that is significantly different from that required for measuring oleic acid content. Table 2 shows the examples of comparative results of defining oleic acid content in sunflower seed oil using chromatographic (GLC) technique and developed method. Measurements were carried out under the conditions close to these of industrial laboratories.

Table 2. Comparative results of defining oleic acid content in sunflower seed oil.

\begin{tabular}{|c|c|c|c|c|c|c|c|c|c|c|c|c|}
\hline \multirow{2}{*}{ Method } & \multicolumn{10}{|c|}{ Oleic acid content in test sample, \% } \\
\cline { 2 - 13 } & 1 & 2 & 3 & 4 & 5 & 6 & 7 & 8 & 9 & 10 & 11 & 12 \\
\hline GLC & 83.9 & 79.0 & 79.6 & 79.6 & 74.8 & 76.0 & 79.7 & 77.7 & 78.4 & 88.5 & 82.3 & 73.1 \\
\hline
\end{tabular}




\begin{tabular}{|c|c|c|c|c|c|c|c|c|c|c|c|c|}
\hline NMR & 74 & 72 & 75 & 72 & 63 & 70 & 69 & 72 & 76 & 78 & 79 & 63 \\
\hline$\Delta$ & 9.9 & 7.0 & 4.6 & 7.6 & 11.8 & 6.0 & 10.7 & 5.7 & 2.4 & 10.5 & 3.3 & 10.1 \\
\hline
\end{tabular}

We should mention that this table shows the results of measuring oleic acid content in seeds that underwent temperature control in laboratory, without measuring their temperature; average value for 5 test samples taken from one sample was considered as the final result. The deviation between 5 dimensions of one sample does not exceed $2 \%$.

Specified data revealed significant differences between results of measuring oleic acid content in seed oil by two different methods. This may be due to various reasons listed below.

It is hard to provide temperature control for each analyzed sample to the required temperature of $23 \pm 0.5^{\circ} \mathrm{C}$ in the conditions of industrial laboratories since up to 300 samples should be analyzed per day. Temperature control of one sample requires at least 2 hours since seed temperature in autumn-winter period differs significantly from the recommended one.

Previous studies have shown that a linear relationship was observed between sample temperature and measured value of the weight content of oleic acid. A temperature change of $1^{\circ} \mathrm{C}$ leads to a $5 \%$ change in oleic acid content measured by NMR. Accordingly, even if sample temperature is determined with an accuracy of $\pm 1^{\circ} \mathrm{C}$, significant errors arise [12].

Oilseeds are complex biological objects. As a result, oleic acid content in them is not constant, even in the seeds grown on the same field under the same conditions. Studies showed that the accumulation of oleic acid in sunflower seed oil can vary significantly even within the seeds of one sunflower head. Therefore, during comparative analyzes, one and the same selected test sample should be tested - not different test samples taken from one sample.

In order to reduce temperature control time of analyzed test samples, it was proposed to use SHF treatment of seeds that was widely used for drying and disinfecting agricultural raw materials. Depending on the selected SHF treatment regimen, its temperature or dehydration may increase [13-15].

A technique was developed for SHF treatment of sunflower seeds. SHF treatment of sunflower seeds allows reducing temperature control time for seeds from 2 hours to 1 minute. This contributes to the quick performance of this analysis.

Studies revealed that optimal conditions of SHF treatment resulted in uniform heating of seeds without loss of moisture what is important in the complex determination of quality parameters of sunflower seeds during their acceptance using pulsed NMR method.

Method of SHF treatment of sunflower seeds is as follows. A test sample of $100 \mathrm{~g}$ should be taken from analyzed seed sample, and its temperature is measured. Depending on the initial temperature, required SHF treatment time is determined according to the calibration graph (Figure 1). Then oil and moisture content should be measured in accordance with the common standard [16] with simultaneous measurement of oleic acid content in sunflower seed oil according to the developed method.

Parameters of SHF treatment may vary and require making calibration graphs depending on the equipment used, in order to find optimal parameters. Sample temperature after SHF treatment is in the range of $20-26^{\circ} \mathrm{C}$. For microwave processing, a household microwave oven (LG MS-20R42D with a capacity of 700W) was used, which allows to reduce the cost of the method's implementation in the conditions of factory laboratories. 




Fig. 1. Dependence of SHF treatment time of a seed sample to a temperature of $23 \pm 0.5^{\circ} \mathrm{C}$ on its initial temperature

To obtain the most accurate results of measuring oleic acid content in sunflower seed oil, the temperature of analyzed test sample should be accurately defined. In the conditions of industrial laboratories, temperature gradient tends to be several degrees what is critical for this method. In order to increase the accuracy of defining sample temperature, a device was developed for sampling sunflower seeds of a given volume of $25 \mathrm{~cm}^{3}$ with a key feature of the ability to measure the temperature of analyzed seed sample immediately before measuring oleic acid content. Physical configuration of this sampling device is shown in Figure 2.

The sampling device includes the following main units: racks of structural aluminum (1), bowl (2), carriage with a dispenser (3), handles for moving the carriage (4), and sample tube retainer (5).

Seed bowl volume is $300 \mathrm{~cm}^{3}$ what makes it possible to take up to 12 test samples with a volume of $25 \mathrm{~cm}^{3}$ from the analyzed sample. At the bottom of the bowl a mount is provided for a seed temperature sensor. Seed bowl is made of plastic what allows reducing the influence of external temperature when measuring the temperature of analyzed sample. The design is quite simple to manufacture due to modern design solutions and adaptive printing methods. To measure the temperature of seeds, a digital sensor is used with an accuracy of temperature measurement of $\pm 0.2^{\circ} \mathrm{C}$. Information about sample temperature is transferred from temperature sensor via USB directly to the computing device of analyzer before each test. 


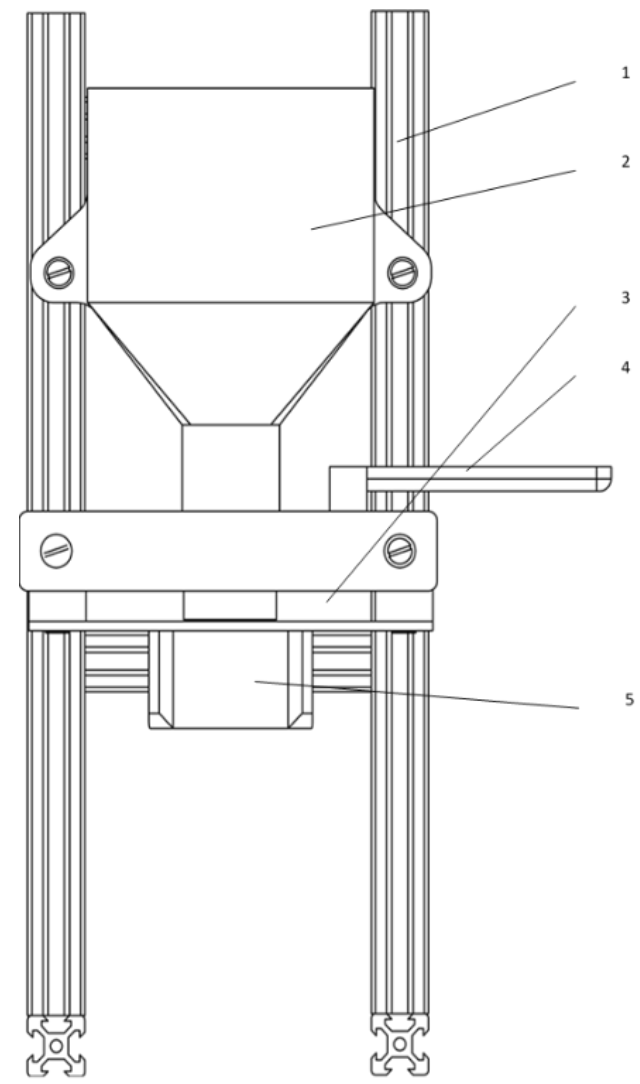

Fig. 2. Sampling device for sunflower seeds.

Table 3 shows the results of measuring oleic acid content in sunflower oil using the developed device for seed dosing with simultaneous measuring of their temperature.

Table 3. Comparative results of defining oleic acid content in sunflower seed oil after improvement.

\begin{tabular}{|c|c|c|c|c|c|c|c|c|c|c|c|c|}
\hline \multirow{2}{*}{ Method } & \multicolumn{10}{|c|}{ Oleic acid content in test sample, \% } \\
\cline { 2 - 15 } & 1 & 2 & 3 & 4 & 5 & 6 & 7 & 8 & 9 & 10 & 11 & 12 \\
\hline GL & 81.1 & 74.9 & 78.7 & 81.7 & 77.0 & 75.5 & 85.6 & 79.3 & 84.5 & 88.1 & 78.0 & 74.6 \\
\hline NMR & 81 & 78 & 81 & 81 & 74 & 77 & 83 & 80 & 82 & 87 & 80 & 74 \\
\hline$\Delta$ & 0.0 & -3.1 & -2.3 & 0.7 & 3.0 & -1.5 & 2.6 & -0.8 & 2.5 & 1.1 & -2.0 & 0.6 \\
\hline
\end{tabular}

We can draw the following conclusions based on data in Table 3. According to the data of Tables 2 and 3, studied seeds showed rather high variability in oleic acid content what should be taken into account during comparative analyzes. Both measurements of oleic acid content were made in the same seed samples prepared for studies one month apart. Average deviation between two replications was $3 \%$ while the maximum one exceeded $6 \%$ for chromatographic method.

It should be mentioned that oleic acid content was initially defined by the developed method based on pulsed NMR technique, and oleic acid content in analyzed samples was determined by chromatographic method. This allowed us to exclude the influence of different quality of samples on the results. 
Measurement of the temperature of each analyzed sample before test and increased number of analyzed samples significantly reduced the error in defining oleic acid content by the developed method (on average, no more than $2 \%$ ).

It should be mentioned that implementation of modern data processing methods reduced test time of one sample. Thus, test time of one sample with doubling the number of analyzed samples did not change significantly what allowed significant reducing the influence of different quality of analyzed samples.

\section{Conclusions}

In the course of our study, it was found that in order to obtain the most accurate results of defining oleic acid content, sample temperature should be $23 \pm 0.5^{\circ} \mathrm{C}$. Moreover, the temperature of each analyzed seed sample should be defined as accurately as it is possible to reduce the influence of temperature factor.

The solution to this problem is a developed sampling device with built-in digital temperature sensor that measures the temperature of each analyzed sample. As a result, error in measuring oleic acid content sunflower seed oil due to the influence of temperature factor was reduced by more than 3 times.

In addition, it is proposed to use microwave processing of sunflower seeds using a household microwave oven with a temperature below $15^{\circ} \mathrm{C}$. This allows to significantly reduce the thermal static time of the sample up to 1 minute. As a result, significantly reduce seed thermostatic time from 2 hours to 2 minutes. The application of the developed technique allows to quickly identify sunflower seeds by the content of oleic acid, while the error of the developed method does not exceed similar values for the GLC method (relative to the discrepancy between the results of the sequential determination, to the average of not more than $7 \%$ ).

\section{References}

1. Ya.N. Demurin, D. Skoric, I. Veresbaranji, S. Jocić, Helia, 23, 87-92 (2000)

2. D. Skoric, S. Jocić, N. Lecic, Z. Sakac, Helia, 30, 205-212 (2007)

3. A. Soolmaz, E. Sattar, A. Abbas, Fatty Acid Compositions and Seed Traits, Helia (2019) doi 10.1515/helia-2019-0009

4. H. Turhan, N. Citak, H. Pehlivanoglu, Z. Mengul, Bulgarian Journal of Agricultural Science, 16, 553-558 (2010)

5. L. V. Zverev et al, Technique. Journal of Analytical Chemistry, 56, 1029-1031 (2001)

6. A. Panyushkin et al, Journal of Applied Spectroscopy - J APPL SPECTROSC, 70, 648651 (2003)

7. A. A. Petrik et al., Method of determining content of oleic acid in sunflower seed oil, Patent RF. no 2241979 (2003)

8. GOST 30418-96, Vegetable oils. Method for determination of fatty acid content. (Minsk, 2013)

9. I. Awatif, S. Arafat, Journal of Food Technology Research, 1 (2), 73-83 (2014)

10. P. Chinachoti, P. Krygsman, Application of Low-Resolution NMR for Simultaneous Moisture and Oil Determination in Food (Oilseeds) (2001) doi 10.1002/0471142913.faa0103s00

11. O.S. Agafonov, S.M. Prudnikov, T.A. Shakhrai, E.P. Victorova, Novye Tehnologii, 4 (50), 11-22 (2019)

12. Device for volumetric dosing of seed samples: patent for utility model 191634 The Russ Federation, IPC G01F 11/40/ O. S. Agafonov, S. M. Prudnikov, -application No. 2019116319; declared 2019.05.27 publ. (14.08.2019) 
13. Y. Kretova, L. Tsirulnichenko, N. Naumenko, N. Popova, I. Kalinina, Agronomy Research, 16 (5), 2079-2087 (2018)

14. M. Keskin, Y. Soysal, Y.E. Sekerli, A. Arslan, N. Celiktas, Agronomy Research, 17 (2), 466-480 (2019)

15. O.V. Mikhailova, M.V. Belova, A.N. Korobkov, G.V. Novikova, VestnikVGUIT [Proceedings of VSUET], 81 (2), 27-34 (2019)

16. GOST 8.597-2010, «State system for ensuring the uniformity of measurements. Oilseeds and oilseeds residues. Determination of oiliness and moisture content using pulsed nuclear magnetic resonance spectrometry» (Moscow, Standartinform Publ., 2011) 